\title{
RADIATION THERAPY- AN EFFECTIVE TOOL FOR ANALGESIA IN METASTATIC BONE DISEASE IN BREAST CANCER
}

\author{
Preety Negi', Pamela Alice Kingsley², Jaineet Sachdeva ${ }^{3}$, Himanshu Srivastava ${ }^{4}$ \\ ${ }^{1}$ Assistant Professor, Department of Radiation Oncology, Christian Medical College \& Hospital, Ludhiana, Punjab, India. \\ 2 Professor and HOD, Department of Radiation Oncology, Christian Medical College \& Hospital, Ludhiana, Punjab, India. \\ 3 Professor, Department of Radiation Oncology, Christian Medical College \& Hospital, Ludhiana, Punjab, India. \\ ${ }^{4}$ Consultant, Department of Radiation Oncology, Capitol Hospital, Jalandhar, Punjab, India.
}

ABSTRACT
BACKGROUND
Radiation therapy plays an important role in the treatment of breast cancer patients with bone metastasis. Approximately $70 \%$ of
patients are likely to achieve complete pain relief after palliative external beam radiotherapy (RT). The aim of current study is to
determine the role of RT in palliation of symptomatic bone metastases from invasive breast cancer.

\section{MATERIALS AND METHODS}

Hundred and twelve breast cancer patients who received palliative RT for bone metastasis were enrolled prospectively. Baseline pain assessment using visual analogue score was done at the time of diagnosis and 3 months following completion of RT. WHO analgesic pain ladder was also used for adequate pain relief. Analgesic requirement was also assessed at the time of diagnosis and 3 months following RT.

\section{RESULTS}

Our results found thoracolumbar spine involvement (87\%) to be the commonest site of metastasis. A reduction in severity of pain was noticed with $79.5 \%$ patients experiencing mild pain, $16 \%$ having moderate pain, and only $3.6 \%$ having severe pain after treatment with palliative RT. Approximately $36.6 \%$ patients experienced significant reduction in analgesic requirement.

\section{CONCLUSION}

This study provides an insight into the combined use of palliative RT and pharmacological treatment for pain relief in breast cancer patients with bone metastasis. We found palliative RT and adequate analgesic use as strong pillars in managing bone metastasis secondary to breast cancer.

\section{KEYWORDS}

Analgesics, Breast Neoplasms, Pain, Pain Management, Retrospective Studies.

HOW TO CITE THIS ARTICLE: Negi P, Kingsley PA, Sachdeva J, et al. Radiation therapy- An effective tool for analgesia in metastatic bone disease in breast cancer. J. Evolution Med. Dent. Sci. 2017;6(61):4450-4453, DOI: 10.14260/Jemds/2017/962

\section{BACKGROUND}

Bone is among the most common metastatic site in breast cancer patients and once distant metastases occur, breast cancer remains a treatable condition but is no longer considered curable. ${ }^{[1,2]}$ Breast cancer patients with bone-only metastasis have a relatively good prognosis as compared to those with extensive disease involving multiple organs.[3,4] Various treatment modalities namely, chemotherapy, hormonal therapy, external beam radiotherapy and bisphosphonate therapy are available therapeutic options for these patients. Bone metastases represent the most frequent indication for palliative radiotherapy (RT) in these patients. The most important goals of palliative RT are pain relief, recalcification and stabilisation, reducing spinal cord compression and minimising the risk of paraplegia. Dose fractionation and the type of RT must be tailored to each

Financial or Other, Competing Interest: None.

Submission 24-06-2017, Peer Review 17-07-2017,

Acceptance 24-07-2017, Published 31-07-2017.

Corresponding Author:

Dr. Preety Negi,

Assistant Professor,

Department of Radiation Oncology,

Christian Medical College \& Hospital,

Ludhiana, Punjab, India.

E-mail: drpreetinegi@gmail.com

DOI: $10.14260 /$ jemds $/ 2017 / 962$ patient individually taking into account the patient's perspective, goals of treatment, and prognosis.[5] Till date, there is a continued debate over the appropriate fractionation scheme of palliative RT to treat these patients. ${ }^{[6]}$ The median survival of patients after development of bone metastasis is approximately two years and $20 \%$ of these patients live longer than 5 years.[7] There is a definite lack of information regarding the best treatment approach for bone metastases leading to prolongation of survival in patients with breast cancer. Early intervention is of paramount importance to control pain and maintain the quality of life. We undertook this study to primarily shed light on the role of palliative RT in relieving pain related to skeletal metastases from breast cancer.

\section{MATERIALS AND METHODS}

The case report forms of all patients treated from 1999 2011 at Christian Medical College \& Hospital, Ludhiana were reviewed.

\section{Inclusion Criteria}

1. Patients with histopathological diagnosis of carcinoma breast with metastatic disease limited to bone.

2. Patients who developed bone metastases as the first site of failure after completing the primary treatment for breast cancer in the form of neoadjuvant/adjuvant 
chemotherapy, surgery with or without external beam radiotherapy to chest wall or intact breast.

\section{Exclusion Criteria}

All patients presenting with treatment failure in the form of visceral metastasis (such as liver, lung or brain).

Diagnosis of bone metastasis was confirmed with whole body bone scintigraphy. Twenty-seven percent patients presented with bone as the first site of failure. The case report form of each patient was scrutinised and demographic details (e.g. age at presentation, menopausal status), clinicopathological data (e.g. T-size, N-size, histology), and treatment details were recorded.

Assessment of the severity of pain was done using Visual analogue score ${ }^{9}$ and it was documented as part of standard institutional protocol in patient record form once on presentation of bone metastasis and at 3 months following completion of palliative RT. After confirmation of bone metastatic disease by whole body bone scintigraphy, all patients received palliative $\mathrm{RT}$ to a dose of 30 Gy in 10 fractions using Cobalt-60 teletherapy unit (Theratron-80R). Radiotherapy planning was done on conventional simulator. All the patients were treated with two field technique i.e. two parallel opposed lateral field. All patients received appropriate pain medications as per the World Health Organization stratified three-step analgesic ladder: Step I using non-opioid analgesics (Acetaminophen or non-steroidal anti-inflammatory drugs- NSAIDS), Step II with "weak" opioids (Hydrocodone, codeine, or tramadol), and Step III with "strong" opioids (Morphine, hydromorphone, oxycodone, fentanyl, or methadone). Additional drugs (Adjuvants) were used as and when required. The patients were moved up or down the ladder based on the clinical assessment of pain. Changes in analgesic requirement preand 3 months post-RT were recorded. Approximately 70\% patients received calcium supplementation.

The data was analysed using SPSS software version 21 . For categorical data, we have calculated $\mathrm{n}(\%)$. A paired t-test was run to determine whether there was a statistically significant mean difference between the pain score and analgesic use before and after the treatment.

\section{RESULTS}

The median age was 51.4 years (range, 25 - 75 years). Majority of patients had advanced T-stage disease (79.5\%) and node-positive disease $(75 \%)$ at presentation. Bone metastasis was present at diagnosis in $27 \%$ patients whereas $73 \%$ patients developed distant metastasis to bone after median follow-up of 54 months. Multiple sites of bone metastases were observed in $77.3 \%$ patients, while the remaining $(22.7 \%)$ had solitary metastases. Among those with multiple bone metastases, spine (59.1\%) was the most frequent site of metastases followed by long bones (40.9\%). Largest number of patients $(87 \%)$ had thoracolumbar spine involvement followed by pelvis (58.9\%), femur (18.5\%), humerus $(13.2 \%)$, sternum $(21.3 \%)$, scapula $(21.5 \%)$ and ribs $(10.7 \%)$ and tibia $(1 \%)$. Solitary metastasis was predominantly seen in the axial skeleton (Table 1).

\begin{tabular}{|c|c|}
\hline Patient Characteristics & No. of Patients ( $n=112)$ \\
\hline \multicolumn{2}{|l|}{ Age (Years) } \\
\hline$\leq 30$ & $5(4.5 \%)$ \\
\hline $31-40$ & $11(9.8 \%)$ \\
\hline $41-50$ & $36(32.1 \%)$ \\
\hline $51-60$ & $46(41.1 \%)$ \\
\hline$\geq 61$ & $14(12.5 \%)$ \\
\hline Mean (range) & 45.7 years \\
\hline \multicolumn{2}{|l|}{ Menopausal Status } \\
\hline Premenopausal & $37(33 \%)$ \\
\hline Postmenopausal & $75(67 \%)$ \\
\hline \multicolumn{2}{|l|}{ ECOG Performance Status } \\
\hline 2 & $34(30.3 \%)$ \\
\hline 3 & $49(43.7 \%)$ \\
\hline 4 & $29(25.9 \%)$ \\
\hline \multicolumn{2}{|l|}{ T-Size } \\
\hline T1 & $2(1.79 \%)$ \\
\hline $\mathrm{T} 2$ & $21(18.8 \%)$ \\
\hline T3 & $57(50.9 \%)$ \\
\hline $\mathrm{T} 4$ & $32(28.6 \%)$ \\
\hline \multicolumn{2}{|l|}{$\mathrm{N}$-Stage } \\
\hline N0 & $28(25 \%)$ \\
\hline N1 & $62(55.4 \%)$ \\
\hline $\mathrm{N} 2$ & $17(15.2 \%)$ \\
\hline N3 & $5(4.5 \%)$ \\
\hline \multicolumn{2}{|l|}{ Stage Grouping } \\
\hline II & $25(22.3 \%)$ \\
\hline III & $57(50.9 \%)$ \\
\hline IV & $30(26.8 \%)$ \\
\hline \multicolumn{2}{|l|}{ Histology } \\
\hline Ductal & $111(99 \%)$ \\
\hline Lobular & $1(1 \%)$ \\
\hline \multicolumn{2}{|l|}{ Type of Lesion } \\
\hline Osteolytic & $65(58 \%)$ \\
\hline Osteoblastic & $25(22.3 \%)$ \\
\hline Both/Mixed & $22(19.6 \%)$ \\
\hline \multicolumn{2}{|l|}{ Receptor Status } \\
\hline ER & Positive in $64 \%$ patients \\
\hline PR & Positive in $50 \%$ patients \\
\hline HER2/neu & - \\
\hline
\end{tabular}

Osteolytic bone metastasis was the most frequently observed radiological pattern accounting for $57.1 \%$. Of 112 patients, $32.1 \%$ had mild pain at presentation, while $50.9 \%$ and $17 \%$ had moderate and severe pain, respectively. Following treatment, the severity of pain decreased with $79.5 \%$ of the patients experiencing no or mild pain, $16 \%$ having moderate pain, and $3.6 \%$ having severe pain (Table 2a). Eight (7.2\%) patients presented with pathological fracture involving humerus and axial skeleton. During followup, $73 \%$ patients developed only bone metastases while $45 \%$ had visceral (lung, liver, brain) metastases.

\begin{tabular}{|c|c|}
\hline VAS before Treatment & No. of Patients \\
\hline $1-3$ (Mild pain) & $36(32.1 \%)$ \\
\hline 4 (Moderate pain) & $57(50.9 \%)$ \\
\hline $5-6$ (Severe pain) & $19(17 \%)$ \\
\hline VAS after treatment & $89(79.5 \%)$ \\
\hline $0-3$ & $18(16 \%)$ \\
\hline 4 & $4(3.6 \%)$ \\
\hline $5-6$ & Table 2a. Pain Scores (VAS) \\
\hline
\end{tabular}




\begin{tabular}{|c|c|c|c|}
\hline & Mean \pm S.D & t-score & p-value \\
\hline VAS before treatment & $1.85 \pm 0.065$ & 13.063 & $0.000^{*}$ \\
\hline VAS after treatment & $1 \pm 0.00$ & & \\
\hline \multicolumn{4}{|c|}{ Table 2b. T-score and Pain scores } \\
\hline
\end{tabular}

VAS after treatment was very mild $(1 \pm 0.00)$ as opposed to VAS before treatment $(1.85 \pm 0.00) ;$ a statistically significant improvement of 0.848 (95\% CI, 0.720 to 0.977 ) in pain score was noticed, $\mathrm{t}(111)=13.063, \mathrm{p}<0.001$ (Table 2b).

As per WHO analgesic ladder, a statistically significant reduction in pain score was observed leading to decrease from $77.7 \%$ to $36.6 \%$ of patients requiring step 2 analgesia (Table 3a).

\begin{tabular}{|c|c|}
\hline Before RT & No. of Patients \\
\hline Step 1 & $20(17.9 \%)$ \\
\hline Step 2 & $87(77.7 \%)$ \\
\hline Step 3 & $5(4.5 \%)$ \\
\hline After RT & $69(61.6 \%)$ \\
\hline Step 1 & $41(36.6 \%)$ \\
\hline Step 2 & $2(1.8 \%)$ \\
\hline Step 3 & Table 3a. WHO Analgesic Ladder Use \\
\hline
\end{tabular}

\begin{tabular}{|c|c|c|c|}
\hline & Mean \pm S.D & T-score & p-value \\
\hline Before RT & $1.87 \pm 0.455$ & 9.474 & $\mathbf{0 . 0 0 0}^{*}$ \\
\hline After RT & $1.40 \pm 0.528$ & & \\
\hline \multicolumn{3}{|c|}{ Table 3b. T-score and Analgesic Use } \\
\hline
\end{tabular}

As per WHO analgesic ladder use, there was a reduction in usage of analgesics after RT $(1.40 \pm 0.528)$ as opposed to before RT $(1.87 \pm 0.455) ;$ a statistically significant improvement of 0.464 (95\% CI, 0.367 to 0.561$)$ in analgesic use was noticed, $\mathrm{t}(111)=9.474, \mathrm{p}<0.001$ (Table $3 \mathrm{~b}$ ).

\section{DISCUSSION}

Breast cancer is now recognised as the most common cancer in Indian women and has overtaken cervical cancer.[8] Approximately $5 \%$ of breast cancer patients have metastatic disease on diagnosis. The bones are the most frequent site of metastatic disease, both at initial diagnosis of disease and as a site of first recurrence.[9,10] We found thoracolumbar spine and pelvis to be the commonest site of bone metastasis similar to as reported in literature.[11]

Bone metastasis is a multistep process involving disturbance between bone formation and resorption as well as the inability of new bone formation in compensating the loss.[12] Imaging modalities such as plain radiography, bone scintigraphy, computed tomography, or magnetic resonance imaging are used for diagnostic workup, allowing a total skeletal assessment for evaluation of the extent of metastatic spread.[13] Majority of our patients were subjected to bone scintigraphy on diagnosis for staging purposes.

Once metastasis occurs, breast cancer is considered a treatable condition but not curable with the exception of micrometastasis.[1,14] Majority of patients with bone metastasis requires an urgent treatment, due to pain, pathological fractures, spinal cord compression, hypercalcaemia and neurologic deficits. ${ }^{[15]}$ Therefore, the aim of palliative RT is to provide complete pain relief, recalcification and stabilisation of the bone, reduction in the risk of complications (e.g. bone fractures, spinal cord compression) and reduce analgesic requirements.[13] Majority of our patients had moderate intensity pain $(50.9 \%)$ with a pain score of 4 while severe pain was encountered by $17 \%$ patients. Pathological fractures commonly occur in patients with bone metastasis from breast cancer and this is mainly due to the lytic nature of bone lesions.[16] In our series, 8 (7.14\%) patients presented with pathological fracture during follow-up.

Patients with the diagnosis of breast cancer with metastatic disease to bone survive longer as compared to those with visceral metastasis. In addition, painful bone metastasis leads to considerable morbidity and reduced quality of life.[17] The aim of treatment for these patients is to provide adequate pain control and good quality of life.[18] Palliative RT can relieve pain in $50-80 \%$ patients achieving complete pain relief in one-third of patients. Unfortunately, the role of palliative RT for alleviation of pain in bone metastatic disease is underutilised. We found palliative RT and adequate analgesic use resulted in improvement with reduction in pain scores in $79.5 \%$ of the patients experiencing no or mild pain, $16 \%$ having moderate pain, and only $3.6 \%$ having severe pain. The rates of pain relief vary from $50 \%$ to $85 \%$ with approximately one-third patients reporting complete response, worldwide. Complete to partial pain relief is said to occur within 4 weeks after RT with a mean duration of remission in 19 weeks.[15] Keeping this in mind, we re-assessed pain scores after 3 months of RT.

There is still a controversy regarding the optimal fractionation schedule of RT in bone metastasis. Multiple randomised controlled data have demonstrated similar pain relief for different regimens, including 30 Gy in 10 fractions, 24 Gy in 6 fractions, 20 Gy in 5 fractions and a single 8-Gy fraction.[19] Until further data are available, it is suggested that short course regimens (i.e. 5 x 4 Gy or 1 x 8 Gy) can be used for patients with short life expectancy, while higher dose per fraction schedules (i.e. 10 × 3 Gy or greater) should be reserved for patients with better prognosis.[13] Rades et al[20] compared the local control of different radiotherapy schedules in patients with metastatic spinal cord compression. A total of 265 patients were randomised to short course ( 1 x 8 Gy or 5 x 4 Gy) versus long course $(10 \times 3$ Gy or $15 \times 2.5 \mathrm{~Gy}$ ) irradiation pattern. Superior 1-year local control and similar survival was reported with long course RT $(61 \%$ versus $81 \% ; p=0.005)$ as compared to short course. The authors concluded that patients with a relatively favourable expected survival should receive long-course RT. Despite various fractionation schedules being used in clinical practice for bone metastasis, the optimal palliative RT fractionation schedule is still controversial. All the patients in our series received palliative RT dose of 30 Gy in 10 fractions over 2 weeks.

We found that the requirement of opioid analgesia dropped from $4.5 \%$ to $1.8 \%$ following palliative RT. In addition, there are reports suggesting that the use of immediate-release morphine before initiating RT allows a higher number of patients to complete the scheduled course of RT.[21]

The present study had limitations of small sample size and bisphosphonates were not given routinely in view of deranged renal parameters, financial constraints, and poor orodental hygiene.

Although the role of palliative RT for bone metastases is well-established in literature, our study provides additional 
information with regard to 30 Gy in 10 fractions being quite effective in achieving symptom control in patients with metastatic bone disease from breast cancer.

\section{CONCLUSION}

Our results indicate that spine and pelvis are the most frequently involved metastatic sites in carcinoma breast. Treating the bone pain caused by cancer is a challenging condition. This study highlights the role of palliative external beam radiotherapy in a dose of 30 Gy in 10 fractions and analgesics in alleviating bone pain associated with bone metastasis in patients with breast cancer with minimal side effects.

\section{ACKNOWLEDGEMENT}

We would like to acknowledge Radiation-Oncology staff for helping with data collection.

\section{REFERENCES}

[1] Cardoso F, Harbeck N, Fallowfield L, et al. Locally recurrent or metastatic breast cancer: ESMO Clinical Practice Guidelines for diagnosis, treatment and follow-up. Ann Oncol 2012;23(7):11-9.

[2] Cardoso F, Costa A, Norton L, et al. 1 ${ }^{\text {st }}$ International Consensus guidelines for advanced breast cancer (ABC 1). Breast 2012;21(3):242-52.

[3] Wong WH, Pavlakis N. Optimal management of bone metastases in breast cancer patients. Breast Cancer 2011;3:35-60.

[4] Coleman RE, Smith P, Rubens RD. Clinical course and prognostic factors following bone recurrence from breast cancer. Br J Cancer 1998;77(2):336-40.

[5] Feyer PC, Steingraeber M. Radiotherapy of bone metastasis in breast cancer patients - Current approaches. Breast Care (Basel) 2012;7(2):108-12.

[6] Lutz S, Berk L, Chang E, et al. Palliative radiotherapy for bone metastasis: an ASTRO evidence-based guideline. Int J Radiat Oncol Biol Phys 2011;79(4):965-76.

[7] Coleman RE. Clinical features of metastatic bone disease and risk of skeletal morbidity. Clinical Care Research 2006;12(20 Pt 2):6243S-9S.

[8] Ghoncheh M, Momenimovahed Z, Salehiniya H. Epidemiology, incidence and mortality of breast cancer in Asia. Asian Pac J Cancer Prev 2016;17(S3):47-52.

[9] Jemal A, Bray F, Center MM, et al. Global cancer statistics. CA Cancer J Clin 2011;61(2):69-90.
[10] Jensen AO, Jacobsen JB, Norgaard M, et al. Incidence of bone metastases and skeletal-related events in breast cancer patients: a population-based cohort study in Denmark. BMC Cancer 2011;11:29.

[11] Kakhki VRD, Anvari K, Sadeghi R, et al. Pattern and distribution of bone metastasis in common malignant tumours. Nuclear Medicine Review Cent East Eur 2013;16(2):66-9.

[12] Yong M, Jensen AO, Jacobsen JB, et al. Survival in breast cancer patients with bone metastases and skeletal-related events: a population-based cohort study in Denmark (1999-2007). Breast Cancer Res Treat 2011;129(2):495-503.

[13] Ripamonti F, Barone G, Maranzano E, et al. Prevention and treatment of bone metastasis in breast cancer. J Clin Med 2013;2(3):151-75.

[14] Pagani O, Senkus E, Wood W, et al. International guidelines for management of metastatic breast cancer: can metastatic breast cancer be cured? J Natl Cancer Inst 2010;102(7):456-63.

[15] De Felice F, Piccioli A, Musio D, et al. The role of radiation therapy in bone metastasis management. Oncotarget 2017;8(15):25691-9.

[16] Singh VA, Haseeb A, Alkubaisi AA. Incidence and outcome of bone metastatic disease at University Malaya Medical Centre. Singapore Med J 2014;55(10):539-46.

[17] Martin M, Bell R, Bourgeois $\mathrm{H}$, et al. Bone-related complications and quality of life in advanced breast cancer: results from a randomized phase III trial of denosumab versus zoledronic acid. Clin Cancer Res 2012;18(17):4841-9.

[18] Zhu YJ. Palliative radiotherapy for painful bone metastases: short course or long-course? Ann Palliat Med 2012;1(1):78-80.

[19] Howell DD, James JL, Hartsell WF, et al. Single-fraction radiotherapy versus multifraction radiotherapy for palliation of painful vertebral bone metastasesequivalent efficacy, less toxicity and more convenient. a subset analysis of Radiation Therapy Oncology Group trial 97-14. Cancer 2013;119(4):888-96.

[20] Rades D, Fehlauer F, Schulte R, et al. Prognostic factors for local control and survival after radiotherapy of metastatic spinal cord compression. J Clin Oncol 2006;24(21):3388-93.

[21] Murino P, Mammucari M, Borrelli D, et al. Role of immediate-release morphine (MIR) in the treatment of predictable pain in radiotherapy. J Pain \& Palliative Care Pharmacotherapy 2011;25(2):121-4. 